Asian J. Med. Biol. Res. 2020, 6 (3), 530-535; doi: 10.3329/ajmbr.v6i3.49805

\author{
Asian Journal of \\ Medical and Biological Research \\ ISSN 2411-4472 (Print) 2412-5571 (Online) \\ www.ebupress.com/journal/ajmbr
}

\title{
Article \\ Management of shifting duties of nurses in a secondary level hospital
}

\author{
Mst. Jannat Ara Ahmed ${ }^{1 *}$, ANM Shamsul Islam², Aseesh Kumar Saha ${ }^{3}$, China Rani Mittra ${ }^{4}$ and Sheuly Begum ${ }^{5}$ \\ ${ }^{1}$ Senior Staff Nurse, 250 Beded General Hospitals, Jashore, Bangladesh \\ ${ }^{2}$ Associate Professor, (NIPSOM), Mohakhali, Dhaka-1212, Bangladesh \\ ${ }^{3}$ Senior Staff Nurse, Upazilla Health Complex, Bagha, Rajshahi, Bangladesh \\ ${ }^{4}$ Senior Staff Nurse, Upazilla Health Complex, Abhaynagar, Jashore, Bangladesh \\ ${ }^{5}$ Senior Staff Nurse, National Institute of Cancer Research \& Hospital, Mohakhali, Dhaka, Bangladesh
}

*Corresponding author: Mst. Jannat Ara Ahmed, Senior Staff Nurse, 250 Bedded General Hospital, Jashore, Bangladesh. Phone: +8801827894589; E-mail: jannatara121@gmail.com

Received: 03 September 2020/Accepted: 24 September 2020/ Published: 30 September 2020

\begin{abstract}
Shifting responsibilities of patient care from one nurse to another is an integral part of nursing practice. There is abundant evidence that inaccurate information in hospital is the primary cause of adverse events and gaps in patient care. A cross-sectional study was conducted in 250 Bedded General Hospital, Jashore from $1^{\text {st }}$ January to $31^{\text {st }}$ December 2017 with the aim to assess the state of nurses shifting duty management. Total 114 nurses were selected conveniently from this hospital and interviewed with a pre-tested semi-structured questionnaire and an observational checklist was used to observe the actual scenario. Data were analyzed by using Statistical Package for Social Science version (SPSS) 24.P-value less than 0.05 were set as statistically significant. Overall shifting duty management of the hospital were categorized into good, average and poor. Among the Nurses overall shifting duty management of the hospital were found as $48.2 \%$ (55) average, $34.3 \%$ (39) poor and $17.5 \%$ (20) good. There was a significant relationship between occurrence of interruption and duration of shifting duty $(\mathrm{p}<0.05)$. Proper staffing and dedicated time management for critical patient's information sharing is needed to reduce gaps in patient care and enhance patient safety thereby. Thus it is essential to establish a standard protocol to ensure proper management of shifting duties of nurses and every hospital should follow this.
\end{abstract}

Keywords: Shifting duty, Shift change, Staffing, Secondary level hospital, Standardized handoff communication, Patient safety.

\section{Introduction}

The word 'shift' means change or exchange in a systematic way and the word 'duty' means task or responsibilities. Thus, the word 'shifting duty' means exchanging responsibilities in a systematic way. Nursing has had a long relationship with duty shifting or duty handover. Nurses shifting duty is a process where one nurse replaces another on the same job within a 24 hour period. Handover is a historic, institutionalized ritual that has remained part of nursing culture throughout the decades. Its roots lie deep in nursing tradition and nursing handover practice continues without questioning its purpose in contemporary times. But this concept of patient hand over is one that has come into sharper focus over the last decade. Vanden (2013) stated that Agency for Healthcare Research and Quality (AHRQ, 2008) identified that the term "hand off" is often used interchangeably with other terms such as handover, sign-out, sign over, cross coverage, and shift report, shift change report etc. (Vanden, 2013). For this study, the term 'shifting duty' was used. Shifting duty is a process involves the transfer of information and responsibility for the care of a patient from one healthcare provider to another. It occurs frequently and at various stages throughout the patient's stay. Change of shift report also occurs between all levels of caregivers from unit aids and nurses to physicians. For the purpose of this paper the focus will be on management of nurses' shifting duty in their unit or ward. 
The nursing change of shift report or handover is a valuable opportunity to transfer responsibility and accountability from one nurse to another in every ward setting of hospitals. It is actually a substantial part of a nurse's duty, and each nurse should allocate $38 \%$ of his or her working hours in a hospital ward every day to fulfill the sign out process (Spanke and Thomas, 2010; Sabet et al., 2015).

The standardization and improvement of the nursing hand off is critical. Nurses are pivotal to ensuring continuity of care since they are accountable for care 24 hours a day, are seen as a key communication partner for all healthcare professionals, and are often the informal coordinators of the increasingly complex care provided in the hospital setting (Smeulers et al., 2014).

The hand off is a traditional part of nursing practice. They stated it as the primary way to exchange information and promote continuity of care for the patient, which may take many different forms varying among institutions, specific units, and even individual nurses. They found nurses use tape recorded, written, or verbal formats to exchange information and these exchanges can occur at the bedside, staff room, or at a central nursing station (O'Connell et al., 2008).

Over the last decade the patients seen in the inpatient care setting have to become increasingly more complex requiring higher levels of care, more complex interventions, and coordination of multiple specialties to manage their disease processes and co-morbidities. This makes effective hand offs between caregivers much more crucial to prevent gaps in care or potential harm to the patient such as medication errors or potential patient death (Friesen et al., 2008).

There was a movement in the developed countries towards standardizing the health sectors by finding each and every single part of its process. Bring change in to shift to shift report communication is also such kind of movement. Cochrane database of systematic reviews in 2014 evidence during the last decade the call for interventions to improve handovers has increased (AHRQ, 2008; Joint Commission, 2012). These interventions aim to reduce the risk of miscommunication, misunderstanding and the omission of critical information; to find out what constitutes an effective nursing handover style (Smeulers et al., 2014).

In developing countries such as Bangladesh, few studies were conducted on the improvement of nursing services, and there is little effort to involve nurses to upgrade their service standards. The first step in standardizing nursing handover and introduction of an alternative model is to improve our understanding of current practices. Although some studies have been conducted in several countries, the handover practices have not been well studied in the Bangladeshi health system because the context is different. Because situational analysis is the first step in changing the program, exploring the current conditions of handover is important. Hence, the aim of this study was to explore the present state of nurses' handover practices in Bangladesh to provide an opportunity for a better understanding of the barriers, facilitators, gaps in patient care due to this process and will help to improve the current practices.

\section{Method and Materials}

\subsection{Ethical Consideration}

The study was conducted with the intension of protecting the human rights of all subjects. At the beginning, formal approval of the study was obtained from the ethical committee of NIPSOM, under the Bangabandhu Sheikh Mujib Medical University (BSMMU), Dhaka, Bangladesh. Before data collection, written permission was obtained from the superintendent of Jashore General Hospital after informing about the purpose of the study. Informed written consent was taken from all respondents.

\subsection{Research Design}

The study was cross sectional study.

\subsection{Study setting}

The study was conducted in 250 Bedded General Hospital, Jashore and it is a Government Hospital.

\subsection{Study Period}

Total Study period was 1 (one) year from $1^{\text {st }}$ January to $31^{\text {st }}$ December 2017.

\subsection{Study Population}

Nurses working in 250 bedded general hospital, Jashore.

\subsection{Sampling technique}

Non-probability purposive sampling technique was used. 


\subsection{Inclusion Criteria}

Nurses who worked in this hospital for more than 6 months and who willingly participated in the study.

\subsection{Tool of the study}

Semi-structured interviewer administered questionnaire and checklist was developed. The questionnaire and checklist was prepared keeping in view of the selected variables according to objectives. The questionnaire was first developed in English version and then translated into Bengali. The questionnaire had two main sections. The information on socio-demographic characteristics of the respondents was included in first section and the information on management of shifting duties of respondents was included in second section.

\section{Results and Discussion}

This cross-sectional study was conducted in a secondary level hospital named 250 Bedded General Hospital, Jashore. The study was carried out among 114 nurses working in nine different wards of this hospital. The demographic characteristics of the respondents are shown in Table 1.

Table 1. Demographic characteristics of respondents by frequency and percentage $(n=114)$.

\begin{tabular}{lll}
\hline Age group & Frequency & Percentage \\
31-35 years & 21 & 18.4 \\
36-40 years & 39 & 34.2 \\
$41-45$ years & 22 & 19.3 \\
46-50 years & 19 & 16.7 \\
51-55 years & 13 & 11.4 \\
Total & 114 & 100.0 \\
Level of education & & \\
Diploma Nursing & 101 & 88.6 \\
BSc Nursing & 13 & 11.3 \\
Length of service in years & Frequency & Percentage \\
5 years and below & 29 & 25.4 \\
6-10 years & 22 & 19.3 \\
11-15 years & 26 & 22.8 \\
16-20 years & 13 & 11.4 \\
21-25 years & 10 & 8.8 \\
26 years and above & 14 & 12.3 \\
Total & $\mathbf{1 1 4}$ & $\mathbf{1 0 0 . 0}$ \\
\hline
\end{tabular}

Table 1 has shown the demographic characteristics of the respondents $(n=114)$. Among 114 respondents above one third [34.2\% (39)] were highest, from 36-40 years age groups and lowest $11.4 \%$ (13) were from 51-55 years age group. The mean age of respondents was $41.46 \pm 6.38$ years. From the respondents $88.6 \%$ (101) were only Diploma in Nursing passed where as very few were passed in BSc in Nursing 6.1\% (7) and BSc in Public Health Nursing 5.3\% (6). Inside the respondents the length of service was highest $25.4 \%$ (29) from 5 years and below, $22.8 \%$ (26) were from 11-15 years and 8.8\% (10) was lowest 21-25 years.

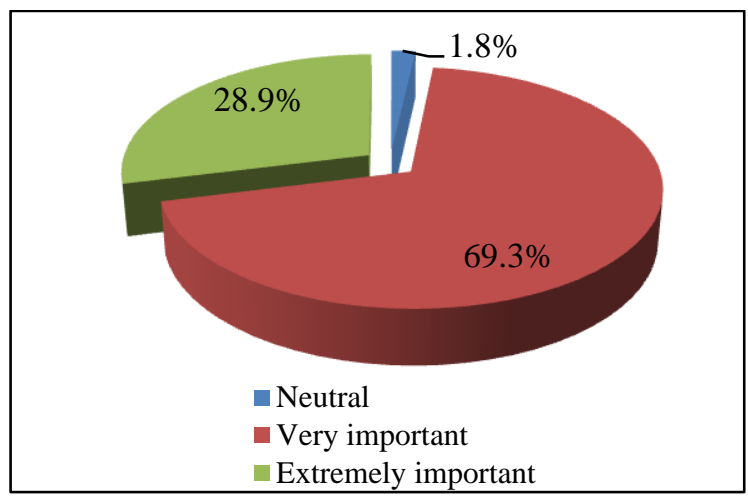

Figure 1. Distribution of respondents regarding importance of shifting duty (n=114). 
Figure 1 demonstrates the importance of shifting duty was thought as very important by $69.3 \%$ (79) of the respondents and $1.8 \%$ (2) considered as neutral.

Table 2. Distribution of respondents by opinion regarding problem and the usual place during shifting duty $(n=114)$.

\begin{tabular}{lll}
\hline Problem during shifting duty & Frequency & Percentage \\
\hline Too little information & 55 & 48.2 \\
Use of confusing language & 47 & 41.2 \\
Time constraints & 12 & 10.5 \\
Total & $\mathbf{1 1 4}$ & $\mathbf{1 0 0 . 0}$ \\
\hline & Usual place & \\
\hline Place of shifting duty & Frequency & Percentage \\
Bed side of the patient & 6 & 5.3 \\
Nurse's station & 77 & 67.5 \\
Only critical patient at bed side and others in the nurse station & 31 & 27.2 \\
Total & $\mathbf{1 1 4}$ & $\mathbf{1 0 0 . 0}$ \\
\hline
\end{tabular}

According to $48.2 \%$ (55) of the respondent's opinion the problem was high regarding too little information during shifting duty among nurses and $10.5 \%$ (12) told about time constraints (Table 2). Beside this, $41.2 \%$ of respondents reported use of confusing language as problem during shifting duty and more than two third of the respondents $[67.5 \%$ (77)] usually shifted their duty at nurses station where as very few [5.3\% (6)] respondents shifted their duty at the bed side of the patients (Table 2).

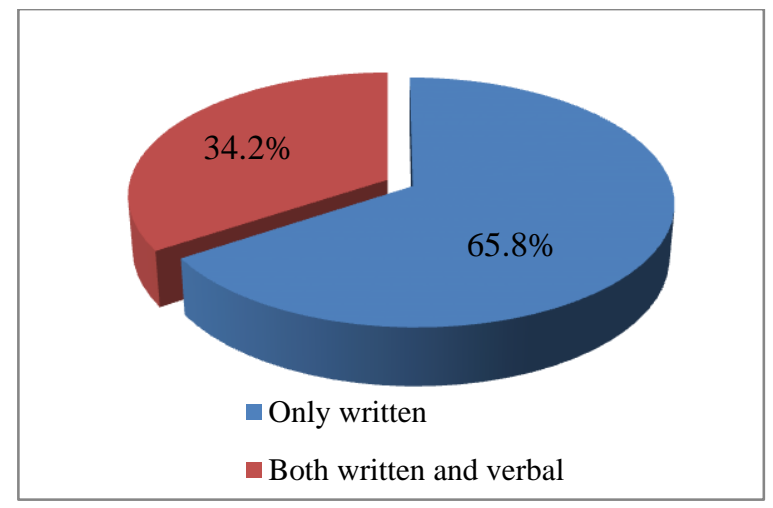

Figure 2. Distribution of respondents by present system of shifting duty (n=114).

Figure 2 represents almost two third of the respondents [65.8\% (75)] followed both written and verbal system of communication during shifting duties and about one third of the respondents [34.2\% (39)] followed only written system of communication.

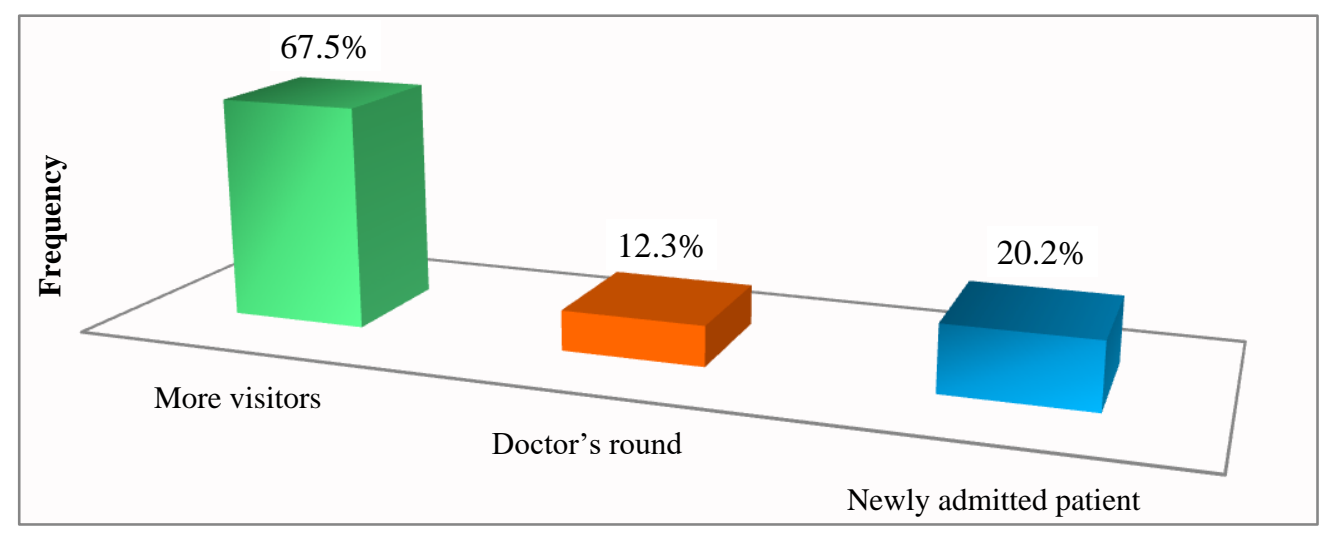

Figure 3. Distribution of respondents regarding types of interruptions $(n=114)$. 
Figure 3 show the above figure demonstrated the type of interruption during shifting duty. Inside the respondents the interruption was $67.5 \%$ due to more visitors and $12.3 \%$ due to doctor's round.

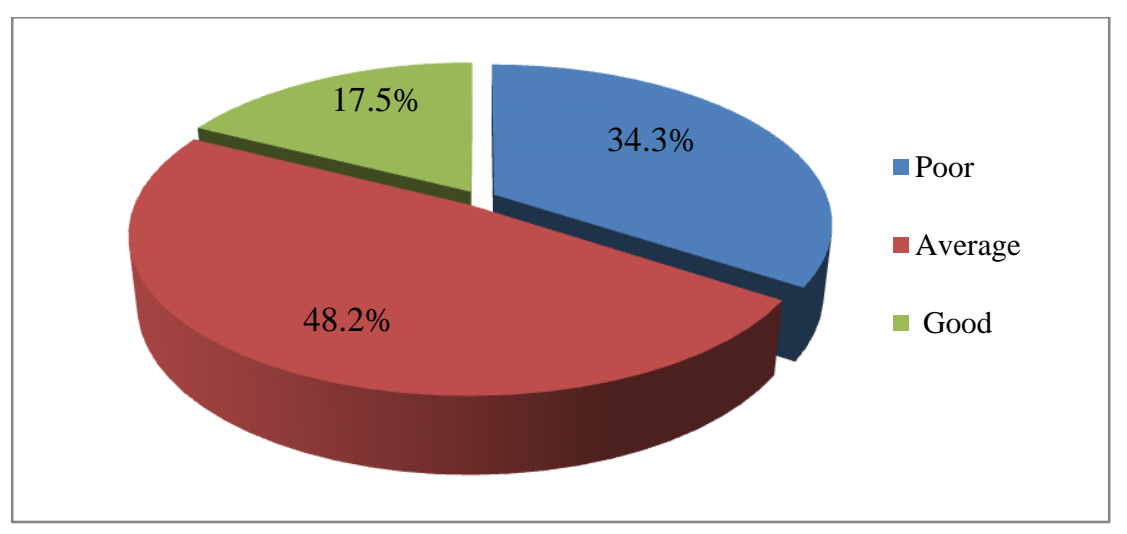

Figure 4. Distribution of the respondents the opinion regarding overall shifting duty management of the hospital (n=114).

Figure 4 describes the opinion regarding overall shifting duty management of the hospital was found as $48.2 \%$ ( 55 ) average, $34.3 \%$ (39) poor and $17.5 \%$ (20) good among the respondents.

Table 3. Association between the occurrence of interruptions during clinical handover and duration of shifting duty $(\mathrm{n}=114)$.

\begin{tabular}{|c|c|c|c|c|}
\hline \multirow{2}{*}{$\begin{array}{l}\text { Occurrence of } \\
\text { interruptions (times) }\end{array}$} & \multicolumn{3}{|c|}{ Usual duration of shifting duty } & Test statistics \\
\hline & Below 5 min $f(\%)$ & $5-10 \min f(\%)$ & $>10 \min f(\%)$ & \multirow{4}{*}{$\begin{array}{l}\chi^{2}=14.302 \\
\mathrm{df}=4 \\
\mathrm{P}=0.006\end{array}$} \\
\hline$\leq 2$ times & $4(8.3 \%)$ & $32(66.7 \%)$ & $12(25.0 \%)$ & \\
\hline 3-4 times & $5(11.9 \%)$ & $21(50 \%)$ & $16(38.1 \%)$ & \\
\hline$\geq 5$ times & $8(33.3 \%)$ & $6(25.0 \%)$ & $10(41.7 \%)$ & \\
\hline
\end{tabular}

Table 3 shows that during clinical handover the occurrence of interruption was less than 2 times among $66.7 \%$ (32) of total respondents with the duration of 5-10 minutes and 8.3\% (4) of respondents with the duration of below 5minutes. The results revealed that there was a significant relationship between occurrence of interruption and duration of shifting duty $(\mathrm{p}<0.05)$.

Table 4. Distribution of the respondents by recommendation regarding improvement of the shifting duty management $(\mathbf{n = 1 1 4})$.

\begin{tabular}{lll}
\hline Recommendations & Frequency & Percentage \\
\hline Computerized system will be better & 8 & 7.0 \\
Follow report khata & 1 & 0.9 \\
Join the duty at right time & 7 & 6.1 \\
Medicine chart need & 13 & 11.4 \\
Need more staff & 29 & 25.4 \\
Need more supervision & 4 & 3.5 \\
Need to be more conscious during shifting duties & 3 & 2.6 \\
Need to be more careful during report writing & 8 & 7.0 \\
Need to be more co-operative & 4 & 3.5 \\
Take sufficient time during shifting duty & 7 & 6.1 \\
Training need for staffs & 21 & 18.4 \\
Visitor restriction during shifting duty & 9 & 7.9 \\
Total & 114 & 100.0 \\
\hline
\end{tabular}

Table 4 represents the suggestions revealed from the respondents regarding the improvement of the shifting duty management. Highest suggestion needed more staff $25.4 \%$ (29) and lowest was follow report khata by $0.9 \%$ (1) of the respondents. 


\section{According to checklist:}

The study revealed that the 250 Bedded General Hospital, Jashore have:

- All the nurses in indoor department were female.

- The shift hand over tool is hand over khata used by all nurses in every ward.

- All respondents were agreed that there is no structured format available for transferring patients from one ward to other ward.

- All respondents were agreed that there is structured format available for referring patients from this hospital to other facilities and they always filled up it.

- All respondents were agreed that there is structured format available for discharging patients from this hospital and they always filled up it.

\section{Conclusions and Recommendations}

The findings regarding the present state of this process does not yield good result and it needs standardization. But standardization does not occur over night. Standardization of handover practices completely depend on the con-text such as culture, philosophy, needs, facilities, priorities, and economic considerations of organization are different from country to country. If the change of shift hand off done properly, can open the door to multiple avenues to improve the quality of patient care; patient safety; relationships between nurses, patients and their families; and also among other nurses.

- A standard operating protocol is recommended to ensure proper management of shifting duties of nurses and every hospital should follow this.

- Nurses should take entry few minutes (15 min.) before the duty time started to minimize the duration of shift overlapping.

- At the end of duty time, nurses should use proper dedicated time for duty shifting to clarify patient's caring pertinent information among them.

- During the active period of shifting duty visitors should be restricted except newly admitted patients.

- Arrangement of proper training, refresher training and practice for nurses on effective shifting duty management should be increased.

\section{Conflict of interest}

None to declare

\section{References}

Agency for Healthcare Research and Quality (AHRQ), 2008. Patient Safety and Quality: An Evidenced-based Practice Handbook for Nurses. Retrieved October 2, 2017 from http://www.ahrq.gov/qual/nurseshdbk/.

Friesen MA, SV White and JF Byers, 2008. Handoffs: implications for nurses. In: Hughes RG, editor. Patient Safety and Quality: An Evidence-Based Handbook for Nurses. Rockville (MD): Agency for Healthcare Research and Quality (US); 2008 Apr.

O’Connell B, C Kelly and K MacDonald 2008. Nursing handover; it's time for a change. Contemp. Nurse, 30: 2-11.

Joint Commission, 2012. Joint Commission introduces new, customized tool to improve hand-off communications. Retrieved $26^{\text {th }}$ August, 2017.

Sabet SR, M Moattari, AN Nasrabadi, M Momennasab and S Yektatalab, 2015. Challenges of nursing handover: A qualitative study. Clin. Nurs. Res., 24: 234-252.

Smeulers M, C Lucas and H Vermeulen, 2014. Effectiveness of different nursing handover styles for ensuring continuity of information in hospitalised patients. Cochrane Database Syst. Rev., 24: CD009979.

Spanke MT and T Thomas, 2010. Nursing assistant walking report at change of shift. J. Nurs. Care Qual., 25: 261-265.

Vanden BAK, 2013. Patient Hand Offs: Facilitating Safe and Effective Transitions of Care. Master's Projects. 1. https://scholarworks.gvsu.edu/kcon_projects/1. 